\title{
Implicit sequence learning is represented by stimulus-response rules
}

\author{
Hillary Schwarb and ERIC H. Schumacher \\ Georgia Institute of Technology, Atlanta, Georgia
}

\begin{abstract}
For nearly two decades, researchers have investigated spatial sequence learning in an attempt to identify what specifically is learned during sequential tasks (e.g., stimulus order, response order, etc.). Despite extensive research, controversy remains concerning the information-processing locus of this learning effect. There are three main theories concerning the nature of spatial sequence learning, corresponding to the perceptual, motor, or response selection (i.e., central mechanisms underlying the association between stimulus and response pairs) processes required for successful task performance. The present data investigate this controversy and support the theory that stimulus-response (S-R) rules are critical for sequence learning. The results from two experiments demonstrate that sequence learning is disrupted only when the S-R rules for the task are altered. When the S-R rules remain constant or involve only a minor transformation, significant sequence learning occurs. These data implicate spatial response selection as a likely mechanism mediating spatial sequential learning.
\end{abstract}

Learning sequences of motor behaviors based on spatial stimuli (e.g., parallel-parking a car, playing the piano, dancing the tango, etc.) is an essential part of our daily lives. It is, therefore, not surprising that the study of sequence learning has been of considerable scientific interest for decades (for reviews, see Cleeremans, Destrebecqz, \& Boyer, 1998; Clegg, DiGirolamo, \& Keele, 1998). Despite the abundance of research, fundamental questions remain about the cognitive processes involved. One unresolved issue involves the information-processing locus of implicit sequence learning. Some theories propose that people learn associations between sequential stimuli, so the learning occurs in relatively early stimulus-encoding processes (stimulus-based theory; e.g., Clegg, 2005; A. Cohen, Ivry, \& Keele, 1990; Howard, Mutter, \& Howard, 1992; Keele, Jennings, Jones, Caulton, \& Cohen, 1995; Mayr, 1996; Stadler, 1989; Verwey \& Clegg, 2005). Other theories propose that people learn the sequence of rules associating stimuli and responses, so the learning occurs within central response selection processes (stimulus-response [S-R] rule theory; see, e.g., Deroost \& Soetens, 2006; Hazeltine, 2002; Schumacher \& Schwarb, 2009; Schwarb \& Schumacher, 2009; Willingham, Nissen, \& Bullemer, 1989). Finally, other theories propose that people learn associations between sequential responses, so the learning occurs in relatively late response execution processes (response-based rule theory; e.g., Bischoff-Grethe, Goedert, Willingham, \& Grafton, 2004; Koch \& Hoffmann, 2000; Willingham, 1999; Willingham, Wells, Farrell, \& Stemwedel, 2000). Here we focus specifically on the S-R rule theory of sequence learning, since we believe that it provides a unifying explanation of the sequence-learning literature.
Since its introduction, the serial reaction time (SRT) task has become a paradigmatic procedure for studying implicit spatial sequence learning (Nissen \& Bullemer, 1987). In the SRT task, participants respond to a target presented in one of several (typically 3-6) possible locations on each trial. Although participants are not told that a sequence exists, the targets are, in fact, presented in a specific sequential order (typically 6-12 positions long) that may be repeated several times throughout a given block of trials. Reaction times (RTs) decrease with practice, presumably (at least in part) because participants benefit from knowledge of the sequence.

Because other factors of general skill learning may also speed RT with practice, many SRT studies measure sequence learning with a transfer effect procedure (see, e.g., A. Cohen et al., 1990; Keele et al., 1995; Willingham et al., 1989). The transfer effect is measured by comparing the mean RTs on a block of random trials (termed the transfer block) with the mean RTs from the preceding and succeeding sequenced-trial blocks. Sequence learning should cause RTs on the sequenced blocks to be significantly faster than those of the intervening random block. This measure is likely to be less affected by ancillary differences in general skill learning because the average amount of general task practice for the sequenced and random blocks is the same.

Thus, the SRT task provides a measure of the extent to which sequence learning has occurred. However, the typical procedure provides little information about what is learned. The studies presented here investigate this question directly and provide support for the S-R rule-based theory of sequence learning. 


\section{Sequence Learning Based on S-R Rule}

In one of the earliest attempts to determine the locus of learning in the SRT task, Willingham et al. (1989, Experiment 3 ) asked participants to respond to the color of an $\mathrm{X}$ (four colors each mapped to separate responses) that appeared at one of four locations. They manipulated whether a sequence occurred in the irrelevant locations, the relevant color stimuli, or neither. The only group to show significant sequence learning (evident by significantly reduced RTs across the training phase) was the one in which the task-relevant stimuli (i.e., the colors) were sequenced. All participants were then switched to the standard SRT task (i.e., participants no longer responded to the color of the stimuli, but rather to their location), and the targets followed the same spatial sequence as during the training phase. Because the $\mathrm{S}-\mathrm{R}$ rules (respond to color vs. respond to location) changed from training to test, during the testing phase there was no benefit of having learned the sequence during the training phase. Willingham and colleagues concluded that learning is neither stimulus based nor response based; rather, sequence learning occurs in the condition-action, or S-R, associations, likely mediated by response selection.

After the theory's introduction (Willingham et al., 1989), the idea that S-R rules are important for sequence learning fell out of favor and the focus of the field shifted. Researchers began to investigate the conditions under which sequence learning did and did not occur (e.g., under dual-task situations, or with variable intertrial intervals; see, e.g., Frensch, Wenke, \& Runger, 1999; Heuer \& Schmidtke, 1996; Shanks \& Channon, 2002; Stadler, 1995), and researchers interested in identifying the locus of learning began to look more specifically at isolating the contribution of stimulus versus response properties on successful sequence learning (e.g., A. Cohen et al., 1990; Howard et al., 1992; Mayr, 1996; Willingham, 1999; Willingham et al., 2000; Ziessler, 1998).

More recently, however, data have been reported that cannot easily be accounted for by either stimulus or response processes alone, and researchers have begun to take a renewed interest in the role of S-R rules in spatial sequence learning. For example, Deroost and Soetens (2006) demonstrated that sequence learning increases with the difficulty of S-R mappings used (i.e., using mappings with arbitrary, or indirect, associations between the stimulus and response, rather than direct ones). The authors proposed that indirect $\mathrm{S}-\mathrm{R}$ mappings require slower, more controlled response selection processes, which facilitate sequence learning. These data support the idea that response selection mediates sequence learning, and sequence learning increases with the duration of response selection.

Additionally, in an fMRI study investigating the neural mechanisms of sequence learning and response selection, Schwarb and Schumacher (2009) demonstrated that manipulations affecting both the amount of sequence learning and response selection difficulty increase activity in largely overlapping neural systems. Furthermore, in a series of dual-task behavioral studies, Schumacher and Schwarb (2009) demonstrated the importance of response selection to the successful learning of spatial sequences. Using a series of dual-task procedures, we showed that sequence learning is impaired only when the central response selection processes of the two tasks proceed in parallel. When response selection stages proceed serially, significant sequence learning occurs, even under dual-task conditions. These data further support the idea that response selection is an important locus of sequence learning.

\section{Alternative Theories of Sequence Learning}

Despite this renewed interest in S-R rules and spatial response selection, two alternative theories of sequence learning have gained substantial support over the last two decades - namely, the stimulus-based and response-based theories of sequence learning. Briefly, the stimulus-based theory of sequence learning suggests that learning is stimulus-specific (Clegg, 2005; Howard et al., 1992; Pashler \& Baylis, 1991), effector-independent (A. Cohen et al., 1990; Keele et al., 1995; Verwey \& Clegg, 2005), nonmotoric (Grafton, Salidis, \& Willingham, 2001; Mayr, 1996), purely perceptual (Howard et al., 1992), and tied to early processing stages (Remillard, 2003, 2009). Conversely, the response-based theory of sequence learning proposes that sequence learning has a motor component and that both making a response and the location of that response are important when learning a sequence (e.g., Bischoff-Grethe et al., 2004; Koch \& Hoffmann, 2000; Willingham, 1999; Willingham et al., 2000), although the motor component may not be the only important factor in sequence learning (Goschke, 1998; Richard, Clegg, \& Seger, 2009).

\section{Attempt to Unify These Theories}

Given the lack of consistency in the theoretical interpretations of sequence-learning research, it may seem daunting to make sense of these various data and theories. However, our research (Schumacher \& Schwarb, 2009; Schwarb \& Schumacher, 2009) and that of others (Deroost \& Soetens, 2006; Hazeltine, 2002; Willingham et al., 1989) propose a unifying principle for these seemingly discrepant results: that the learning effect is mediated by the association of S-R rules in response selection. In fact, the $\mathrm{S}-\mathrm{R}$ rule theory of sequence learning can explain and reinterpret many of the findings supporting alternative theories in the literature (see the General Discussion section).

Some findings, however, remain inconsistent with the $\mathrm{S}-\mathrm{R}$ rule theory. In particular, the $\mathrm{S}-\mathrm{R}$ rule account does not offer a simple explanation for the data reported by Willingham (1999, Experiment 3). Using the SRT task, participants in Willingham's study were trained and showed significant sequence learning with a sequence requiring indirect manual responses. This indirect mapping required participants to respond with the button one position to the right of the target (except for the rightmost response; see Figure 1A). After training, participants switched to a direct S-R mapping (shown in Figure 1B). During the testing phase, either the sequence of responses (response-constant group) or the sequence of stimuli (stimulus-constant group) was maintained. As shown by 
A

Indirect Mapping

Willingham (1999, Experiment 3)

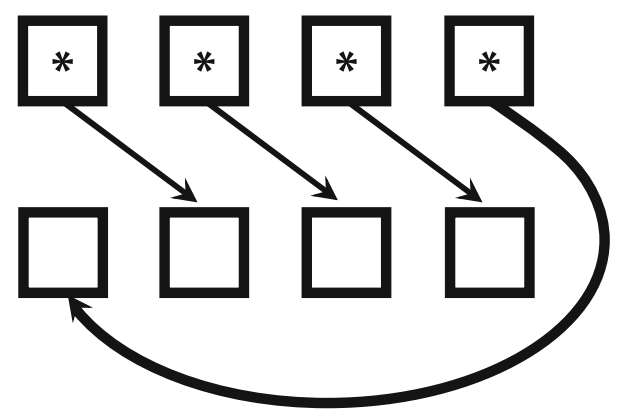

B Direct Mapping
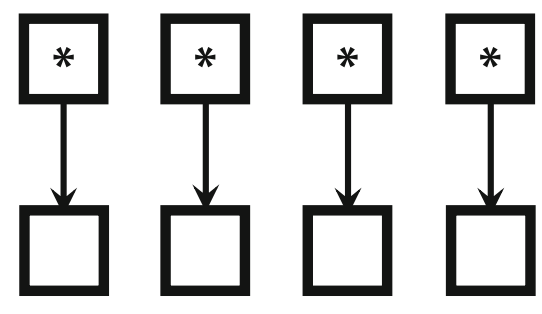

C

Indirect Mapping

Experiment 1

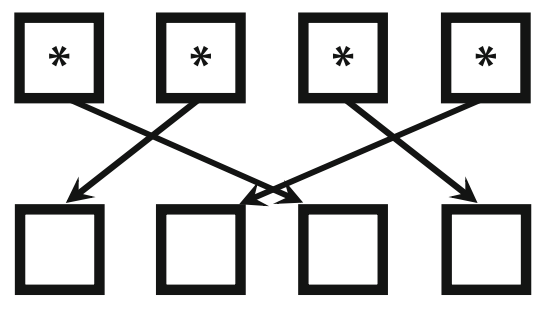

Figure 1. Indirect and direct $S-R$ mappings. The squares containing asterisks are the possible target locations; the empty squares represent the response buttons pressed by participants' left middle, left index, right index, and right middle fingers. The arrows indicate which manual response should be made to each target. (A) Indirect $\mathbf{S}-\mathbf{R}$ mappings used both in Willingham (1999, Experiment 3) and the present Experiment 2. (B) Direct $S-R$ mapping used in both Willingham's experiments and the present ones. (C) Indirect $S-R$ mapping used in Experiment 1.

the striped bars in Figure 3, the response-constant group, not the stimulus-constant group, showed significant learning. Because maintaining the sequence structure of the stimuli from training phase to testing phase did not facilitate sequence learning but maintaining the sequence structure of the responses did, Willingham concluded that response processes (i.e., learning of response locations) mediate sequence learning.

According to the $\mathrm{S}-\mathrm{R}$ rule theory, changing the $\mathrm{S}-\mathrm{R}$ rules from training to test (i.e., from the $\mathrm{S}-\mathrm{R}$ mapping shown in Figure 1A to the one shown in Figure 1B) in the response-constant group should have disrupted sequence learning. To investigate this challenge to the S-R rule theory, the present experiments tested an alternative account for Willingham's (1999) findings. The present research replicates and extends the results from Willingham's Experiment 3 and proposes a potential resolution: localizing sequence learning in response selection processes mediating $\mathrm{S}-\mathrm{R}$ rule association. We hypothesized that the particular change in S-R mappings used by Willingham did not substantially alter the underlying $\mathrm{S}-\mathrm{R}$ rules. Under this hypothesis, participants' performance in the two experimental phases was not contingent on two sets of S-R rules, but a simple transformation of the originally learned $\mathrm{S}-\mathrm{R}$ rules (i.e., shift response one position to the left). Thus, the $\mathrm{S}-\mathrm{R}$ rules learned during training were able to facilitate learning in the response-constant group during the testing phase.

\section{EXPERIMENT 1}

Experiment 1 was designed to directly evaluate the hypothesis that sequence learning involves response selection. Experiment 1 closely replicated the Willingham (1999) experiment with a modified indirect S-R mapping (shown in Figure 1C) that was not a simple transformation of the direct mapping, therefore likely requiring a different set of S-R rules during the training and testing phases (e.g., Duncan, 1977; Kornblum, Hasbroucq, \& Osman, 1990). We hypothesized that when these S-R rules were maintained across the experiment, participants would show a benefit of sequence knowledge. Conversely, when only the stimulus sequence or the response sequence was maintained (i.e., stimulus-constant and response-constant groups, respectively), sequence knowledge would not benefit performance during the testing phase.

\section{Method}

\section{Participants}

One hundred two participants from the Georgia Institute of Technology participated in this study in partial fulfillment of a course requirement. None of the participants was aware of the purpose of the study. Participants gave written informed consent prior to beginning the experiment.

\section{Stimuli and Apparatus}

Stimuli presentation and RT measurements were implemented using E-Prime software (Schneider, Eschman, \& Zuccolotto, 2002) running on a Dell Dimension 3000 PC with a 17 -in. CRT monitor. Manual responses were made with the four outer buttons of a Psychology Software Tools serial response box, and participants viewed the visual display from a distance of approximately $60 \mathrm{~cm}$.

\section{Design and Procedure}

SRT task. Four evenly spaced squares outlined in white on a black background were presented horizontally in the center of the computer monitor. This display remained on the screen throughout the experiment. Each square subtended $2^{\circ}$ of visual angle on each side, and the center-to-center distance between squares was $5^{\circ}$ of visual angle. The entire horizontal display subtended $17^{\circ}$ of visual angle. The target was an asterisk, which subtended $0.3^{\circ}$ of visual angle and appeared in the center of one of the squares on each trial.

The experiment was divided into two phases (shown in Table 1): a training phase (Blocks 1-8) and a testing phase (Blocks 9-12). The training phase consisted of 8 blocks of 96 trials each. Each block presented either an untrained sequence (new sequence on every block) or a trained sequence (same sequence repeated in several blocks). In this experiment, untrained sequences were used in place of random 
trials. These untrained sequences were matched to the trained sequences for ancillary differences that have been shown to influence task performance (cf. Reed \& Johnson, 1994). During the training phase, an untrained sequence was used in Blocks 1 and 8 , and the trained sequence was used in Blocks 2-6. The testing phase consisted of 4 blocks of 96 trials each. During the testing phase, the trained sequence was used only in Block 11, and an untrained sequence was used in Blocks 9, 10, and 12. The block order and logic of the testing phase were identical to those in the testing phases of similar studies (e.g., Willingham, 1999, Experiments 1 and 2; Willingham et al., 2000). These studies have suggested that two blocks using an untrained sequence preceding one block using a trained sequence are necessary in order to calculate an accurate transfer effect not confounded by general performance adjustments. Previous data (e.g., Willingham, 1999, Experiment 3) have suggested that the block directly following a mapping change is unstable and, more generally, not an accurate reflection of task performance. Thus, in this design Block 9 may involve additional processes necessary for participants to adjust to changes in the S-R mapping (described below). Therefore, the second block was used to calculate the transfer effect. The entire experiment consisted of 12 blocks and 1,152 trials.

S-R mapping. There were two S-R mappings used in this experiment. When using the direct S-R mapping (Figure 1B), participants responded to the targets from left to right with their left middle, left index, right index, and right middle fingers, respectively. When using the indirect S-R mapping (Figure 1C), participants responded to the targets from left to right with their right index, left middle, right middle, and left index fingers, respectively. All participants used the direct $\mathrm{S}-\mathrm{R}$ mapping during the testing phase. Training phase mapping was contingent on group.

Groups. Participants were randomly assigned to three groups: the S-R constant, stimulus-constant, and response-constant groups. The $\mathrm{S}-\mathrm{R}$ constant group used the direct $\mathrm{S}-\mathrm{R}$ mapping in both the training and the testing phases. The stimulus-constant group used the indirect $\mathrm{S}-\mathrm{R}$ mapping during the training phase and the direct S-R mapping during the testing phase. Although the mapping changed, the stimulus presentation order remained the same across the entire experiment. The response-constant group also used the indirect S-R mapping during the training phase and the direct $\mathrm{S}-\mathrm{R}$ mapping during the testing phase. For this group, although the mapping changed, the response order remained the same across the entire experiment.

Procedure. For all groups, the four squares appeared on the screen at the start of each trial and remained on-screen for the duration of the trial. When a trial began, a target appeared in one of the four locations and remained on-screen until a response was made. Participants were instructed to respond to the targets as quickly and as accurately as possible using their left and right middle and index fingers. The next trial began $250 \mathrm{msec}$ after a response had been made. When an error was made, an $800-\mathrm{Hz}$ tone sounded for $125 \mathrm{msec}$ during the 250 -msec response-stimulus interval. S-R mappings varied between training and testing phase for the three groups as described above.

Mean RT and accuracy was presented to each participant at the end of each block. At that time participants were encouraged to respond as quickly and accurately as possible in the upcoming block.

Sequences. Six sequences were used in this experiment. The sequences followed the statistical rules defined by Reed and Johnson
(1994). As is shown in Table 1, one of the sequences was repeated in each of the blocks using the trained sequence (Blocks 2-7 and 11 ), and a different sequence was used in each of the blocks using an untrained sequence (Blocks 1, 8-10, and 12). Sequences were counterbalanced across participants.

Practice. Before the start of the experiment, participants completed four ( $\mathrm{S}-\mathrm{R}$ constant group) or five (stimulus-constant and response-constant groups) practice blocks. These practice blocks were methodologically identical to the experimental blocks, except that the target positions were always random and the blocks consisted of 40 trials each. Also, accuracy feedback was presented between each trial, whereas both accuracy and RT feedback were presented at the end of each block. The S-R constant group completed four blocks of trials using the direct $\mathrm{S}-\mathrm{R}$ mapping (used during both the training and testing phases). The stimulus-constant and responseconstant groups completed one block of trials using the direct S-R mapping (used during the testing phase) and four blocks of trials using the indirect $\mathrm{S}-\mathrm{R}$ mapping (used during the training phase). Participants in the stimulus-constant and response-constant groups received more practice than did participants in the S-R constant group because two separate S-R mappings (direct and indirect) were required of these participants and so they practiced both, whereas the $\mathrm{S}-\mathrm{R}$ constant group used and practiced only the direct S-R mapping. Practice was designed so that all participants received an equal number of practice blocks using the $\mathrm{S}-\mathrm{R}$ mapping required during the training phase where sequence knowledge was acquired.

Explicit knowledge/free-generation test. After the experiment, all participants completed a free-generation task similar to one used by Willingham (1999) to measure their explicit knowledge of the sequences. Before the free-generation task, participants were informed that the targets followed a sequence. They were then asked to reproduce the sequence by pressing the response keys. When a button was pressed, the corresponding target appeared on the screen. Participants were permitted to make up to 26 responses.

\section{Results}

\section{Explicit Knowledge/Free-Generation Test}

The free-generation test was scored as in Willingham (1999). A free-generation score (the sum of all correct responses) was calculated for each participant. Not all correct responses had to be recalled consecutively, but the correct response did have to be a part of a segment of at least three consecutive positions. For example, if the sequence were 3-4-3-1-2-4-1-3-2-1-4-2 (with each digit representing a target appearing at one of the four spatial locations) and a participant produced the sequence 3-1-2-3-1-4-2 on the free-generation test, the participants' score would be 6 , because 3-1-2 and 1-4-2 are both part of the original sequence. A given portion of the sequence was scored only once. Therefore, if a participant produced the sequence 3-4-3-1, that participant's score would be 4, because although 3-4-3 and 4-3-1 are both correct sequences of three consecutive positions, the 4-3 portion was counted

Table 1

Training Phase and Testing Phase in Experiment 1

\begin{tabular}{lllllllllllll}
\hline & \multicolumn{10}{c}{ Block } \\
\cline { 2 - 12 } & 1 & 2 & 3 & 4 & 5 & 6 & 7 & 8 & 9 & 10 & 11 & 12 \\
\hline Block type & UT & T & T & T & T & T & T & UT & UT & UT & T & UT \\
Sequence type & S1 & S2 & S2 & S2 & S2 & S2 & S2 & S3 & S4 & S5 & S2 & S6 \\
Phase & Tr & Tr & Tr & Tr & Tr & Tr & Tr & Tr & Te & Te & Te & Te \\
\hline
\end{tabular}

Note- $\mathrm{T}$, trained sequence; UT, untrained sequence; $\mathrm{Tr}$, training phase; Te, testing phase. Blocks 7 ,

$8,10,11$, and 12 refer to the transfer effect. 
only once, so the maximum score on the free-generation task was 12. Mean recall scores were 7.7, 6.2, and 5.8 for the $\mathrm{S}-\mathrm{R}$ constant, stimulus-constant, and response-constant groups, respectively. A one-way ANOVA was conducted and the main effect of group was significant $[F(2,97)=$ $3.45, p=.036]$. An alpha level of .05 was used for all statistical tests. Post hoc analysis revealed that the S-R constant group had significantly higher free recall scores than did the response-constant group ( $p=.045)$. The stimulusconstant group was not significantly different from either the response-constant or the $\mathrm{S}-\mathrm{R}$ constant groups ( $p=$ 1.00 and $p=.178$, respectively).

\section{Participant Selection}

It has been suggested that implicit and explicit learning are fundamentally different mechanisms $(\mathrm{N}$. J. Cohen \& Eichenbaum, 1993; Reber, Allen, \& Reber, 1999) and are mediated by different cortical processing systems (Clegg et al., 1998; Keele, Ivry, Mayr, Hazeltine, \& Heuer, 2003; Reber et al., 1999), so including participants who show evidence of explicit knowledge in an analysis can dramatically affect the results (Willingham, 1999). Furthermore, Willingham (Experiment 1) demonstrated that responsebased learning may be specific to the implicit knowledge domain. Therefore, so as to most directly evaluate each theory of sequence learning (i.e., stimulus-based, response-based, or S-R rule-based), only participants with low levels of explicit knowledge (i.e., scores of less than 10 on the free-generation test, as in Willingham, 1999) were included in the analysis. Seventeen participants (10 from the $\mathrm{S}-\mathrm{R}$ constant group, 3 from the stimulus-constant group, and 4 from the response-constant group) were thus removed from the analysis.

Additional participants were removed from the analysis if their training phase transfer effect was more than 2.5 standard deviations below the mean. As a result, 1 additional participant was removed from each of the S-R constant and the response-constant groups. Participants demonstrating training phase accuracy scores that were more than $2.5 S D \mathrm{~s}$ below the mean were also removed from the analysis. As a result, 1 additional participant was removed from each of the S-R constant and the stimulus-constant groups. RT trials that were $2.5 \mathrm{SDs}$ above or below the mean were then removed from each experimental block of trials. Removing outliers reduced the variability in the data but did not alter the pattern of results. Finally, participants eliminated from the study were replaced to ensure equal group sizes (27 participants in each group).

\section{RTs: Training Phase Sequenced Blocks}

Mean RT data were analyzed with a two-way ANOVA with a between-subjects variable for group (S-R constant, stimulus constant, response constant) and a within-subjects variable for block (2-7). The test for sphericity was significant $(p=.001)$, so the degrees of freedom were corrected according to the Huynh-Feldt adjustment. As is shown in the top panel of Figure 2, the ANOVA revealed a significant main effect of both block $[F(4.6,355.1)=46.47, p<$ $.001]$ and group $[F(2,78)=70.10, p<.001]$, as well as a significant block $\times$ group interaction $[F(9.1,355.1)=$ $7.81, p<.001]$. Mean RTs for both the stimulus-constant and response-constant groups decreased substantially with practice, whereas mean RTs for the S-R constant group decreased to a lesser extent.

\section{Training Phase Transfer Effect}

If sequence learning occurred during the training phase, we would expect RTs to change as the sequence structure changed (the trained sequence to an untrained sequence); that is, mean RT from Block 8 should be slower than the mean RT from Block 7. Because of the specificity of this transfer effect prediction, one-tailed comparisons were used in all significance tests. As is shown in the bottom panel of Figure 2, mean RTs significantly increased after switching from Block 7 to Block 8 for the S-R constant $[27 \pm 3 \mathrm{msec} ; t(26)=9.23, p<.001]$, stimulus-constant $[41 \pm 19 \mathrm{msec} ; t(26)=2.12, p=.021]$, and responseconstant [ $35 \pm 11 \mathrm{msec} ; t(26)=3.25, p=.002]$ groups. These transfer effect results indicate that participants in all three groups acquired knowledge of the sequence during the training phase.

A two-way repeated measures ANOVA with group (S-R constant, stimulus constant, and response constant) as a between-subjects variable and structure (Block 7 [trained sequence], Block 8 [untrained sequence]) as a withinsubjects variable was conducted on these training phase transfer data. The main effect of structure was significant $[F(1,78)=21.36, p<.001]$, indicating that participants were faster on Block 7 than on Block 8. The main effect of group was also significant $[F(2,78)=60.70, p<.001]$, indicating that the $\mathrm{S}-\mathrm{R}$ constant group was faster than the other two groups. The structure $\times$ group interaction was not significant $[F(2,78)=0.28, p=.754]$.

\section{Testing Phase Transfer Effect}

Transfer effects were investigated using one-tailed $t$ tests comparing the mean RT from Block 11, which used the trained sequence, and the average mean RTs from the surrounding untrained sequence blocks (Block 10 and Block 12). As is shown in the bottom panel of Figure 2, the testing phase transfer effect was significant for the $\mathrm{S}-\mathrm{R}$ constant group $(17 \pm 4 \mathrm{msec} ; t(26)=3.96, p<.001]$ and was not significant for either the stimulus-constant $(-2 \pm 5 \mathrm{msec}$; one-tailed $t$ test not appropriate) or the response-constant $[3 \pm 4 \mathrm{msec} ; t(26)=0.74, p=.235]$ group.

A two-way structure $\times$ group ANOVA was also performed on the testing phase transfer data. The main effect of structure $[F(1,78)=5.35, p=.023]$, the main effect of group $[F(2,78)=7.20, p=.001]$, and the structure $\times$ group interaction $[F(2,78)=4.45, p=.015]$ were all significant. Post hoc analysis revealed that the transfer effect RTs for the S-R constant group were significantly larger than the transfer effect RTs for both the stimulus-constant $(p=.004)$ and response-constant $(p=.005)$ groups. The stimulus-constant and response-constant groups were not significantly different from each other $(p=1.00)$. These analyses show that only the $\mathrm{S}-\mathrm{R}$ constant group showed a significant effect of learning at test. 

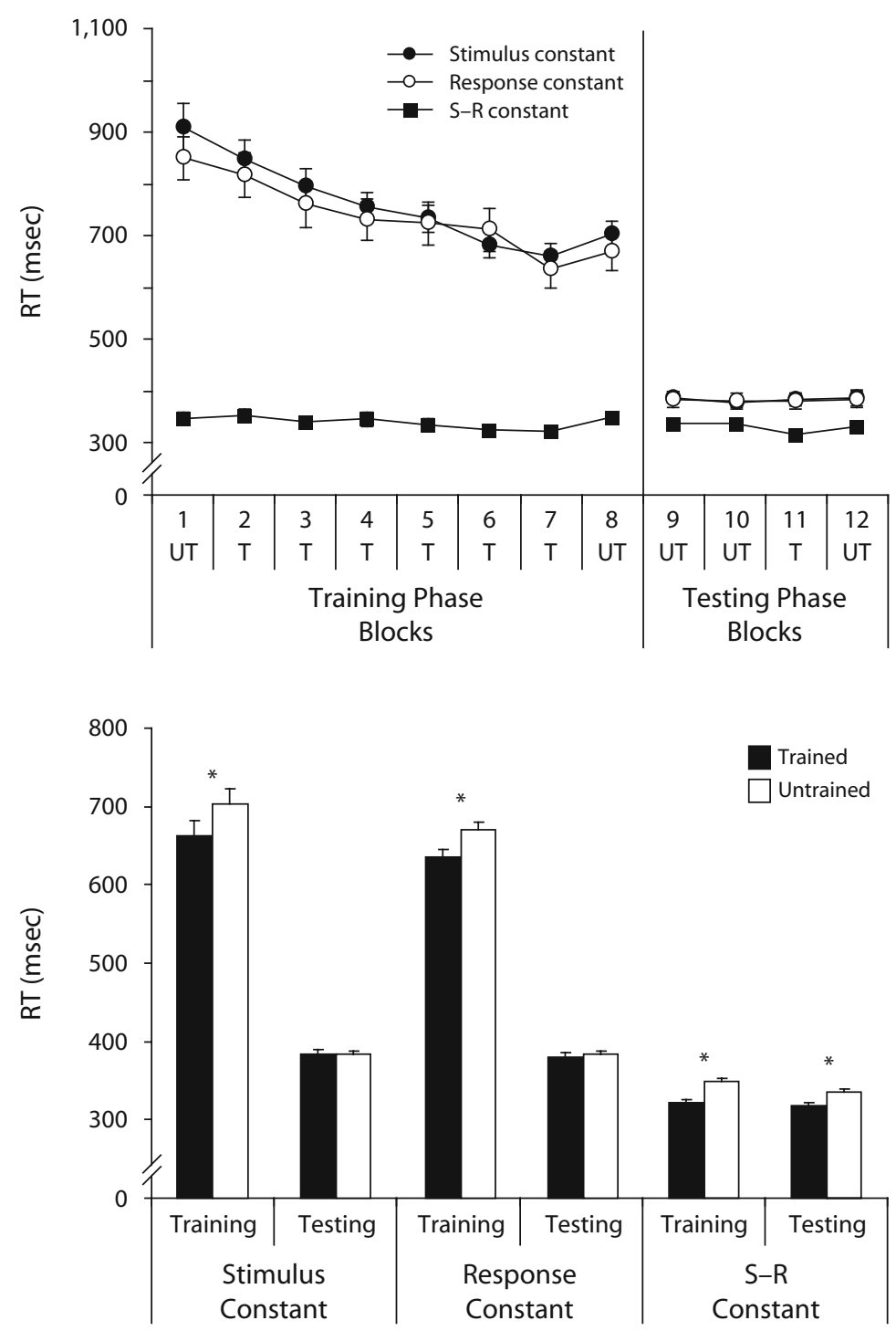

Figure 2. Top panel: Mean reaction times (RTs) and standard errors across blocks for stimulus-constant, response-constant, and stimulus-responseconstant ( $S-R$ constant) groups in Experiment 1. Bottom panel: Mean RTs and standard errors for the blocks using trained and untrained sequences to calculate the transfer effect in both the training and testing phases of Experiment 1. Data are presented for each of the three experimental conditions (stimulus constant, response constant, and $S-R$ constant). ${ }^{*} p<.05$, one-tailed.

\section{Error Rates}

Mean training phase error rates were $2.2 \%, 2.7 \%$, and $2.7 \%$ for the S-R constant, stimulus-constant, and response-constant groups, respectively. An arcsine transformation $\left(p^{\prime}=\arcsin \downarrow p\right.$ ) was performed to stabilize the variance of the error rates (Kleinbaum, Kupper, Muller, \& Nizam, 1998). These data were submitted to a twoway ANOVA with a between-subjects variable for group ( $\mathrm{S}-\mathrm{R}$ constant, stimulus constant, and response constant) and a within-subjects variable for block (2-7). The test for sphericity was not significant $(p=.129)$, so sphericity was assumed. Neither the main effect of block $[F(5,390)=1.01, p=.371]$ nor the block $\times$ group inter- action $[F(10,390)=0.85, p=.582]$ was significant. The main effect of group $[F(2,78)=4.67, p=.012]$ was significant. Post hoc analysis of group differences revealed that accuracy for the stimulus-constant and responseconstant groups did not differ $(p=1.00)$, but the $\mathrm{S}-\mathrm{R}$ constant group was significantly more accurate than either the stimulus-constant $(p=.025)$ or the responseconstant $(p=.035)$ group. ${ }^{1}$

For the training phase, transfer effect error rates were $3.0 \%, 4.4 \%$, and $3.4 \%$ for the S-R constant, stimulusconstant, and response-constant groups, respectively. These error rates were also analyzed. Training phase transfer effects were not significant for the stimulus- 
constant $[-0.1 \% \pm 2.7 \% ; t(26)=-0.59, p=.558]$ or the response-constant $[-0.2 \% \pm 2.4 \% ; t(26)=-1.22$, $p=.232]$ groups. The $\mathrm{S}-\mathrm{R}$ constant group did show a significant transfer effect $[0.9 \% \pm 2.9 \% ; t(26)=2.19, p=$ $.038]$. Block 7 (trained sequence) was significantly more accurate than Block 8 (untrained sequence).

For the testing phase, transfer effect error rates were $2.5 \%, 1.8 \%$, and $2.0 \%$ for the S-R constant, stimulusconstant, and response-constant groups, respectively. Testing phase transfer effects were also not significant for the stimulus-constant group $[-0.5 \% \pm 1.7 \% ; t(26)=-0.89$, $p=.383]$. The testing phase transfer effect was significant for both the response-constant $[0.7 \% \pm 1.7 \% ; t(26)=2.32$, $p=.029]$ and the $\mathrm{S}-\mathrm{R}$ constant $[0.4 \% \pm 1.8 \% ; t(26)=$ $2.23, p=.034]$ groups again, with participants performing more accurately on blocks using the trained sequence than blocks using an untrained sequence.

\section{Discussion}

Results from Experiment 1 demonstrate that, although participants in all the groups acquired sequence knowledge during the training phase, sequence knowledge was evident during the testing phase only when the sequence of S-R rules remained constant between both phases of the experiment. These data represent a failure to replicate Willingham (1999, Experiment 3), and are inconsistent with both the stimulus- and response-based accounts of sequence learning. If stimulus-stimulus associations were learned during the training phase through early stimulus-encoding processes (e.g., A. Cohen et al., 1990; Howard et al., 1992; Mayr, 1996), participants in the stimulus-constant group should have shown a significant transfer effect during the testing phase when the sequence of stimuli was maintained. Similarly, if response-response associations or response locations mediate sequence learning through response execution processes (Willingham, 1999), the response-constant group should have shown learning during the testing phase when the sequence of responses was maintained.

We acknowledge here that the interesting result of Experiment 1 partially rests on a null finding - a lack of transfer effect in two of the three experimental groups (although the structure $\times$ group interaction was significant). This issue will be addressed in the Discussion following Experiment 2.

The failure of Experiment 1 to replicate Willingham (1999, Experiment 3) was predicted by the S-R rule theory outlined in the introduction. The indirect $\mathrm{S}-\mathrm{R}$ mapping used in the training phase of the present study was composed of a novel set of S-R associations compared with the direct S-R mapping used in the testing phase (Duncan, 1977; Kornblum et al., 1990; Read \& Proctor, 2004). Therefore, unlike participants in Willingham's experiment, the present participants could not use a simple transformation of the $\mathrm{S}-\mathrm{R}$ rules learned during training (e.g., shift response one position to the left and wrap around last response) to support performance during the testing phase.

The results from Experiment 1 (i.e., the failure to replicate Willingham, 1999, Experiment 3) are consistent with the $\mathrm{S}-\mathrm{R}$ rule theory of sequence learning. However, other ancillary differences between these experiments may account for the discrepant results. The effect of these differences was investigated in Experiment 2.

\section{EXPERIMENT 2}

The main procedural difference between Experiment 1 and Willingham's (1999, Experiment 3) study was the indirect S-R mapping used. However, other minor alterations were also included (e.g., additional blocks using the trained sequence during the training phase to increase learning; an additional block using an untrained sequence at the start of the testing phase to stabilize performance prior to measuring the transfer of sequence knowledge; sequences conforming to the rules described by Reed \& Johnson, 1994; and possibly other differences in participants and/or procedural implementation because Experiment 1 was conducted 10 years after Willingham's [1999] experiment).

Although there is little theoretical reason to think that these changes could produce the discrepant results, we addressed this possibility directly in a second experiment. Experiment 2 is an exact replication of the major experimental design of Willingham (1999, Experiment 3) with the minor procedural design changes from the present Experiment 1. That is, this experiment is an exact replication of Experiment 1, except that the indirect S-R mapping from Willingham (our Figure 1A) was used instead of the indirect $\mathrm{S}-\mathrm{R}$ mapping from Experiment 1 (Figure 1C). Therefore, if the present Experiment 2 replicates the results from Willingham's Experiment 3, we can be confident that the change in S-R mapping explains the difference between the results from the present Experiment 1 and the results from the present Experiment 2 and Willingham's Experiment 3.

\section{Method}

\section{Participants}

Fifty-one naive participants from the Georgia Institute of Technology participated in this study in partial fulfillment of a course requirement. As in Experiment 1, none of the participants was aware of the purpose of the study. Participants gave informed consent prior to beginning the experiment.

\section{Stimuli and Apparatus}

The stimuli and apparatus were as in Experiment 1.

\section{Design and Procedure}

SRT task. The SRT task was as in Experiment 1, except that only stimulus-constant and response-constant groups were included.

Procedure. The procedure was identical to that of Experiment 1 except that the indirect S-R mapping from Willingham (1999) was used (Figure 1A). During indirect mapping trials, participants responded using the key one position to the right of the target location, with a far-right target "wrapping around" to require a far-left response. Thus, participants responded to the targets from left to right with their left index, right index, right middle, and left middle fingers, respectively.

Sequences. The sequences were the same as those used in Experiment 1.

Practice. The practice was identical to that in Experiment 1, except that the new indirect $\mathrm{S}-\mathrm{R}$ mapping was used during indirect practice blocks. 


\section{Results}

\section{Explicit Knowledge/Free-Generation Test}

The free-generation test was scored as in Willingham (1999) and Experiment 1. Mean recall scores were 7.2 and 5.8 for the stimulus-constant and response-constant groups, respectively. A one-way ANOVA was conducted, and the main effect of group was not significant $[F(1,49)=$ $2.68, p=.108]$.

\section{Participant Selection}

As in Experiment 1,9 participants (5 from the stimulusconstant group and 4 from the response-constant group) demonstrated particularly high free-generation scores (10 or greater) and were removed from the analysis.

One additional participant from the stimulus-constant group was removed from the analysis because training phase learning scores were more than two $S D$ s below the mean. As in Experiment 1, RT trials that were 2.5 SDs above or below the mean were removed from each experimental block, and analyses were conducted on these reduced data. Again, trends in the original data and the data after outliers were removed were similar.

\section{RTs: Training Phase Sequenced Blocks}

As in Experiment 1, mean RT data were analyzed with a two-way ANOVA with a between-subjects variable for group (stimulus constant and response constant) and a within-subjects variable for block (2-7). The test of sphericity was significant $(p<.001)$, so the degrees of freedom were corrected according to the Huynh-Feldt adjustment. The ANOVA revealed a significant main effect of block $[F(3.1,122.7)=17.43, p<.001]$. Neither the main effect of group $[F(1,39)=0.50, p=.485]$ nor the block $\times$ group interaction $[F(3.1,122.7)=0.28$, $p=.851]$ was significant. These data are plotted in Figure 3.

\section{Training Phase Transfer Effect}

Training phase transfer effects were investigated comparing Block 7 (trained sequence) with Block 8 (untrained sequence) and were analyzed using one-tailed comparisons. As is shown in Figure 3, mean RTs significantly increased after switching from Block 7 to Block 8 for both the stimulus-constant $[23 \pm 11 \mathrm{msec} ; t(19)=$ 2.23, $p=.019]$ and response-constant [36 $13 \mathrm{msec}$; $t(20)=2.82, p=.006]$ groups. These significant transfer effect results indicate that participants in both groups acquired knowledge of the sequence during the training phase.

A two-way repeated measures ANOVA with group (stimulus constant and response constant) as a betweensubjects variable and structure (Block 7 [trained sequence] and Block 8 [untrained sequence]) as a within-subjects variable was then conducted on these data. The main effect of structure was significant $[F(1,39)=12.77, p=.001]$, indicating that participants were faster on Block 7 than on Block 8. Neither the main effect of group $[F(1,39)=$ $1.06, p=.309]$ nor the structure $\times$ group interaction $[F(1,39)=0.64, p=.427]$ was significant.

\section{Testing Phase Transfer Effect}

Testing phase transfer effects were calculated comparing the mean RT from Block 11, which used the trained sequence, and the average mean RTs from the surrounding blocks (Blocks 10 and 12), which each used an untrained sequence (Figure 3). The testing phase transfer effect was significant for the response-constant group $[19 \pm 8 \mathrm{msec} ; t(20)=2.42, p=.016]$ but not for the stimulus-constant group $(-3 \pm 7 \mathrm{msec}$; one-tailed $t$ test not appropriate).

A two-way structure $\times$ group ANOVA was also performed on the testing phase transfer data. Both the main effect of structure $[F(1,39)=2.58, p=.116]$ and the main effect of group $[F(1,39)=0.89, p=.352]$ were not significant. The structure $\times$ group interaction reached significance $[F(1,39)=4.35, p=.044]$. This interaction indicates that the size of transfer effect was different for the two groups (i.e., larger for the response-constant than for the stimulus-constant groups).

\section{Error Rates}

Mean training phase error rates were 3.6\% and 3.0\% for the stimulus-constant and response-constant groups, respectively. As in Experiment 1, an arcsine transformation $\left(p^{\prime}=\arcsin \sqrt{ } p\right.$ ) was performed to stabilize the variance of the error rates (Kleinbaum et al., 1998) and a two-way ANOVA with a between-subjects variable for group (stimulus constant, response constant) and a within-subjects variable for block (2-7) was performed. The test for sphericity was not significant $(p=.389)$, so sphericity was assumed. Neither the main effect of block $[F(5,195)=0.33, p=.893]$, the main effect of group $[F(1,39)=0.73, p=.400]$, nor the block $\times$ group interaction $[F(5,195)=0.40, p=.846]$ was significant.

Transfer effect error rates were $3.5 \%$ and $3.1 \%$ for the stimulus-constant and response-constant groups, respectively. These data were also analyzed. Training phase transfer effects were not significant for either the stimulusconstant $[0.5 \% \pm 3.4 \% ; t(19)=-0.56, p=.581]$ or the response-constant $[0.2 \% \pm 2.9 \% ; t(20)=0.13, p=.897]$ groups. Testing phase transfer effects were not significant for the stimulus-constant $[-0.2 \% \pm 2.1 \% ; t(19)=1.22$, $p=.237]$ or the response-constant $[0.4 \% \pm 1.7 \% ; t(20)=$ $0.58, p=.572]$ groups.

\section{Discussion}

The data from Experiment 2 directly replicate the findings from Willingham (1999, Experiment 3). Sequence knowledge was not evident at test when the S-R mapping changed, but the sequence of stimulus locations was maintained. However, when the sequence of response locations was maintained across the experiment despite the S-R mapping change, sequence learning endured. These data indicate that the novel finding from Experiment 1 (i.e., no evidence of sequence knowledge for the response-constant group at test) is likely the result of the indirect $\mathrm{S}-\mathrm{R}$ mapping used in that experiment and not the more subtle differences in the procedure used by Willingham. 

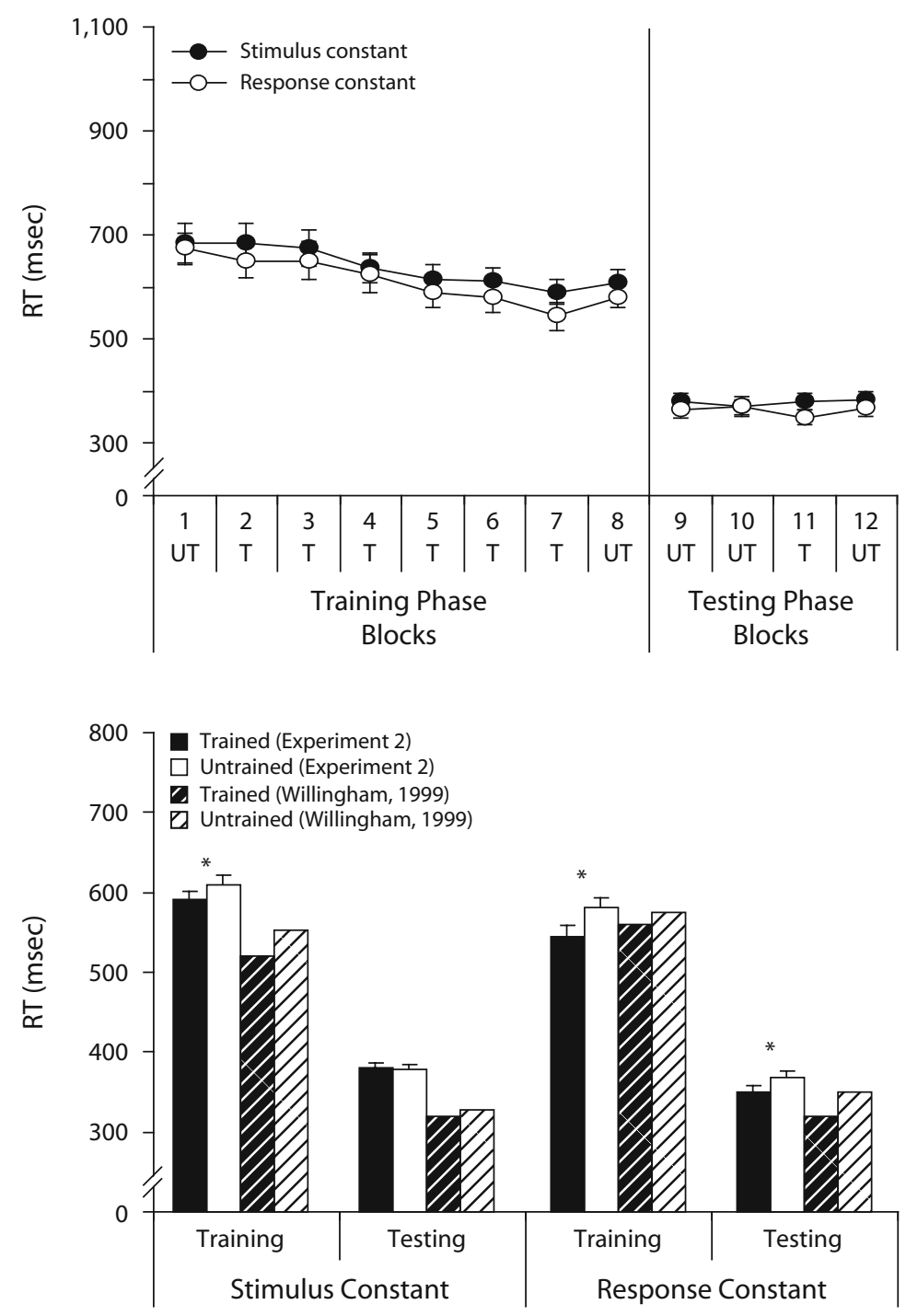

Figure 3. Top panel: Mean reaction times (RTs) and standard errors across blocks for both stimulus-constant and response-constant groups in Experiment 2. Bottom panel: Solid bars indicate mean RTs and standard errors for the blocks using trained and untrained sequences to calculate the transfer effect in both the training and transfer phases of Experiment 2. Data are presented for both experimental conditions (stimulus constant and response constant). For comparison purposes, hatched bars represent an estimation of the comparable experimental conditions (i.e., stimulus constant and response constant) in Willingham (1999, Experiment 3). Significant effects for the present data are indicated. ${ }^{*} p<.05$, one-tailed.

Furthermore, we were able to directly assess the effect of the differences in indirect $\mathrm{S}-\mathrm{R}$ mappings between the two experiments by conducting a two-way repeated measures structure $\times$ experiment ANOVA on the responseconstant group testing phase data. In this analysis, the structure $\times$ experiment interaction $[F(1,46)=3.82, p=$ $.057]$ approached significance, indicating a difference between the response-constant groups' testing phase transfer effects between the two experiments. Thus, although the interesting result for the response-constant group in Experiment 1 was based on a null effect (i.e., no transfer effect during the testing phase of the experiment), this between-experiments interaction suggests that the difference in S-R mappings used in each experiment produced meaningfully different results. Thus, our mapping manipulation was effective in altering learning in the SRT task. We believe that this difference is due to the $\mathrm{S}-\mathrm{R}$ rule requirements in each experiment.

\section{GENERAL DISCUSSION}

In the present experiments, we evaluated the role of S-R rule association and response selection in sequence learning. These experiments are an extension of the Willingham 
(1999, Experiment 3) findings previously reported. Experiment 1 tested the prediction that if the $\mathrm{S}-\mathrm{R}$ rules required in the training phase were substantially altered in the testing phase, there would be no evidence of sequence learning, regardless of whether the stimulus sequence or the response sequence was maintained. This prediction was supported by the data. Only when the S-R rules were held constant across the experiment was there evidence for sequence learning at test. Experiment 2 replicated the Willingham finding using his direct and indirect S-R mappings, while integrating minor alterations to the experimental design so as to be consistent with the procedure of Experiment 1 . Consistent with Willingham's data, we found that when the mapping was altered but the response sequence maintained, sequence learning was evident at test. This replication adds support and consistency to the existing literature, while providing an interesting and important comparison group for the data from Experiment 1.

Data from the two present experiments together support the hypothesis that, in an SRT task, participants learn a sequence by acquiring knowledge of the rules that bind stimulus and response representations. These data demonstrate the importance of response selection in the learning of spatial sequences (Deroost \& Soetens, 2006; Hazeltine, 2002; Schumacher \& Schwarb, 2009; Schwarb \& Schumacher, 2009; Willingham et al., 1989). This finding is important, because it both explains an inconsistency between the predictions of the S-R rule theory and the existing literature and offers a new interpretation of existing sequence-learning data. To date, the sequence-learning literature has been riddled with inconsistent findings and opposing interpretations. This controversy is fueled by inconsistent data supporting various theories. We proposeand the present data support - a possible unifying theory that can explain the discrepancies between stimulusbased and response-based accounts of sequence learning; namely, sequence learning is mediated by response selection, a central component of SRT learning.

The present results add to a growing body of literature supporting the idea that spatial response selection plays an important role in the learning of spatial sequences (e.g., Deroost \& Soetens, 2006; Hazeltine, 2002; Schumacher \& Schwarb, 2009; Schwarb \& Schumacher, 2009; Willingham et al., 1989). In order for an appropriate response to be made, it has been proposed that task-relevant S-R pairs must be activated in working memory (Curtis \& D'Esposito, 2003; Miller \& Cohen, 2001; Pashler, 1994; Rowe, Toni, Josephs, Frackowiak, \& Passingham, 2000; Schumacher, Cole, \& D'Esposito, 2007; Schumacher \& Schwarb, 2009). These $\mathrm{S}-\mathrm{R}$ pairs remain in working memory across several trials, thus allowing associations between the active S-R pairs to develop and learning to occur (N. J. Cohen \& Eichenbaum, 1993; Frensch, Buchner, \& Lin, 1994).

Additionally, it has been previously noted that the importance of response selection in sequence learning logically implicates the role of S-R rules (Duncan, 1977). However, in understanding the importance of $\mathrm{S}-\mathrm{R}$ rules, a critical distinction must be made. We hypothesize that it is the $\mathrm{S}-\mathrm{R}$ rule set and not merely individual $\mathrm{S}-\mathrm{R}$ as- sociations that are essential for learning a sequence. We believe that what Willingham (1999; Willingham et al., 2000) investigated was the effect of S-R associations: very specific stimuli (e.g., a shaded circle or a digit) paired with very specific responses (e.g., a particular key or location). Duncan, on the other hand, demonstrated that S-R mappings are governed by systems of rules and not individual $\mathrm{S}-\mathrm{R}$ associations, and that these rules could be applied to numerous $\mathrm{S}-\mathrm{R}$ associations. Thus, in a four-position direct S-R mapping task, in which participants respond with the button positioned directly below the target location (e.g., Bischoff-Grethe et al., 2004; Olson et al., 2006; Willingham, 1999; Willingham et al., 2000), one S-R rule may describe all four $\mathrm{S}-\mathrm{R}$ associations. This distinction explains the response-constant group data in Willingham (1999, Experiment 3) and in the present Experiment 2. When the mapping was altered from training to test, participants could apply a transformation of the governing $\mathrm{S}-\mathrm{R}$ rules to the seemingly new $\mathrm{S}-\mathrm{R}$ associations. For the more complex S-R mappings used in Experiment 1, however, participants were unable to apply simple rules, and had to rely on individual S-R associations (Read \& Proctor, 2004).

Studies reporting that sequence learning is effectorindependent (A. Cohen et al., 1990; Keele et al., 1995; Verwey \& Clegg, 2005) can easily be explained within this S-R rule framework. When participants learn a sequence with, for example, three-finger responses, they learn a set of $\mathrm{S}-\mathrm{R}$ rules. When knowledge of the sequence is tested with, for example, one-finger responses (A. Cohen et al., 1990), this changes the response execution processes, not response selection (i.e., the $\mathrm{S}-\mathrm{R}$ rules do not change). Similarly, when participants learn a sequence with one hand and then switch to the other hand (see, e.g., Verwey \& Clegg, 2005), this switch requires only a simple transformation of the previously learned S-R rules.

S-R rule theory can also explain the results that were previously thought to support a response-based theory of sequence learning. Willingham (1999, Experiment 1), for example, reported that when participants only watch the series of stimuli (cf. Howard et al., 1992), learning does not occur (provided that participants were unaware of the sequence), but that when participants respond to those stimuli, they learn the sequence. According to the S-R rule account, participants who only observe the sequence do not require and therefore may not learn S-R rules during observation, so they do not learn the sequence.

Furthermore, other literatures report data using tasks other than the SRT task that provide additional support for the S-R rule theory. Pashler and Baylis (1991), for example, demonstrated that when digits, letters, and symbols were mapped onto three buttons from left to right (training phase) and different digits, letters, and symbols were later mapped onto those three buttons (testing phase), learning endured (Experiment 1). If, during the testing phase, the same stimuli were remapped as letters-symbols-digits from left to right (i.e., the category-response mapping was altered), this change disrupted performance (Experiment 4). Finally, if participants continued to respond to digits-letters-symbols from left to right during the testing 
phase but switched hands, performance persisted (Experiment 5). Together, these data suggest that only when the $\mathrm{S}-\mathrm{R}$ rules were maintained and S-R rules not disrupted did participants show learning during the testing phase.

Although the conclusions of the present study provide a clear and comprehensive framework for understanding many of the discrepant results reported in the literature, several limitations to the theory must be noted. First, it has been demonstrated that participants are sometimes able to learn two separate sequences presented simultaneously, one sequence of stimuli and one sequence of responses (e.g., Goschke, 1998). For example, Goschke dissociated stimulus order from response order in a task that presented four letters on the computer screen as well as an auditory cue on each trial. The participants then had to find the visually presented letter indicated by the auditory cue and to respond to the location of that letter. Both the presentation of auditory cues and the required response order comprised separate and independent sequences. Using this procedure, Goschke demonstrated that participants were able to learn both the sequence of auditory cues and the sequence of required responses simultaneously. The $\mathrm{S}-\mathrm{R}$ rule theory is not able to explain these data, since the S-R rules change on every trial. However, the Goschke findings are inconsistent with other studies in which participants fail to learn simultaneously presented sequences using tasks that are more similar to the standard SRT task (e.g., Mayr, 1996; Schmidtke \& Heuer, 1997). Data such as these may call into question the generalizability of results from SRT-type tasks to implicit sequence learning more generally. Unfortunately, theoretical accounts from the SRT task are not commonly applied or investigated using other tests of implicit learning, so the generalizability of the findings reported here is unknown. This is an important question that requires further investigation.

Finally, in addition to those theories of sequence learning investigated in the present experiments, there is an additional theory that deserves mention. The response-effect theory of sequence learning (e.g., Ziessler, 1998; Ziessler \& Nattkemper, 2001) states that response-stimulus (R-S) learning is a major mechanism in successful sequence learning. This theory suggests that when participants are presented with a spatial sequence, learning and performance improvements depend on learning the relationship between a current response and the following target location (Ziessler, 1998; Ziessler \& Nattkemper, 2001). Much data in support of this theory come from the serial search-and-reaction task, in which various letters are presented in a $5 \times 5$ matrix. Letter stimuli and button push responses are organized in an eight-to-four S-R mapping, so that the relationship between response and upcoming target location can be systematically varied. Data from this procedure demonstrate a performance improvement when a response predicts the following target location, even when the presentation order of stimuli and required responses is random (Ziessler, 1998). Unfortunately, the data presented in the present studies are unable to address this theory directly, because, with the one-to-one stimulus response mapping used here, $\mathrm{R}-\mathrm{S}$ order is confounded with both the stimulus and response sequence. Therefore, it remains possible that $\mathrm{R}-\mathrm{S}$ learning also plays a critical role in spatial sequence learning in the SRT task. Further research and clever task manipulations will be necessary to investigate the role of R-S learning in the SRT task.

In conclusion, the present study sought to identify the information-processing locus of spatial sequence learning. Our data suggest that when learning a spatial sequence, individuals learn a series of S-R rule associations. Learning transfers when the same or similar S-R rules can be used during training and testing phases. This $\mathrm{S}-\mathrm{R}$ rule theory provides a unifying account of much of the discrepant findings in the spatial sequence-learning literature and offers a framework, implicating response selection, for providing a more consistent and coherent account of the mechanisms underlying spatial sequence learning.

\section{AUTHOR NOTE}

Correspondence concerning this article should be addressed to H. Schwarb or E. H. Schumacher, School of Psychology, Georgia Institute of Technology, 654 Cherry Street, Atlanta, GA 30332-0170 (e-mail: hschwarb@gatech.edu or eschu@gatech.edu).

\section{REFERENCES}

Bischoff-Grethe, A., Goedert, K. M., Willingham, D. T., \& Grafton, S. T. (2004). Neural substrates of response-based sequence learning using fMRI. Journal of Cognitive Neuroscience, 16, 127138. doi:10.1162/089892904322755610

Cleeremans, A., Destrebecqz, A., \& Boyer, M. (1998). Implicit learning: News from the front. Trends in Cognitive Sciences, 2, 406416. doi:10.1016/S1364-6613(98)01232-7

Clegg, B. A. (2005). Stimulus-specific sequence representation in serial reaction time tasks. Quarterly Journal of Experimental Psychology, 58A, 1087-1101. doi:10.1080/02724980443000485

Clegg, B. A., Digirolamo, G. J., \& Keele, S. W. (1998). Sequence learning. Trends in Cognitive Sciences, 2, 275-281. doi:10.1016/ S1364-6613(98)01202-9

Cohen, A., Ivry, R. I., \& Keele, S. W. (1990). Attention and structure in sequence learning. Journal of Experimental Psychology: Learning, Memory, \& Cognition, 16, 17-30. doi:10.1037/0278-7393.16.1.17

Cohen, N. J., \& Eichenbaum, H. (1993). Memory, amnesia, and the hippocampal system. Cambridge, MA: MIT Press.

Curtis, C. E., \& D'Esposito, M. (2003). Persistent activity in the prefrontal cortex during working memory. Trends in Cognitive Sciences, 7, 415-423. doi:10.1016/S1364-6613(03)00197-9

Deroost, N., \& Soetens, E. (2006). The role of response selection in sequence learning. Quarterly Journal of Experimental Psychology, 59, 449-456. doi:10.1080/17470210500462684

Duncan, J. (1977). Response selection rules in spatial choice reaction tasks. In S. Dornic (Ed.), Attention and performance VI (pp. 49-61). Hillsdale, NJ: Erlbaum.

Frensch, P. A., Buchner, A., \& Lin, J. (1994). Implicit learning of unique and ambiguous serial transitions in the presence and absence of a distractor task. Journal of Experimental Psychology: Learning, Memory, \& Cognition, 20, 567-584. doi:10.1037/0278 $-7393.20 .3 .567$

Frensch, P. A., Wenke, D., \& Runger, D. (1999). A secondary tonecounting task suppresses expression of knowledge in the serial reaction task. Journal of Experimental Psychology: Learning, Memory, \& Cognition, 25, 260-274. doi:10.1037/0278-7393.25.1.260

Goschке, T. (1998). Implicit learning of perceptual and motor sequences: Evidence for independent systems. In M. A. Stadler \& P. Frensch (Eds.), Handbook of implicit learning (pp. 401-444). Thousand Oaks, CA: Sage.

Grafton, S. T., Salidis, J., \& Willingham, D. B. (2001). Motor learning of compatible and incompatible visuomotor maps. Journal of Cognitive Neuroscience, 13, 217-231. doi:10.1162/089892901564270 
Hazeltine, E. (2002). The representational nature of sequence learning: Evidence for goal-based codes. In W. Prinz \& B. Hommel (Eds.), Common mechanisms in perception and action: Attention and performance XIX (pp. 673-689). Oxford: Oxford University Press.

Heuer, H., \& SchmidtKe, V. (1996). Secondary-task effects on sequence learning. Psychological Research/Psychologische Forschung, 59, 119-133. doi:10.1007/BF01792433

Howard, J. H., MutTer, S. A., \& Howard, D. V. (1992). Serial pattern learning by event observation. Journal of Experimental Psychology: Learning, Memory, \& Cognition, 18, 1029-1039. doi:10.1037/0278 -7393.18.5.1029

Keele, S. W., Ivry, R., Mayr, U., Hazeltine, E., \& Heuer, H. (2003). The cognitive and neural architecture of sequence representation. Psychological Review, 110, 316-339. doi:10.1037/0033 $-295 X .110 .2 .316$

Keele, S. W., Jennings, P., Jones, S., Caulton, D., \& Cohen, A. (1995). On the modularity of sequence representation. Journal of Motor Behavior, 27, 17-30.

Kleinbaum, D. G., Kupper, L. L., Muller, K. E., \& Nizam, A. (1998). Applied regression analysis and other multivariable methods (3rd ed.). Pacific Grove, CA: Thomson Brooks/Cole.

Koch, I., \& Hoffmann, J. (2000). Patterns, chunks, and hierarchies in serial reaction-time tasks. Psychological Research, 63, 22-35. doi:10.1007/PL00008165

Kornblum, S., HasbroucQ, T., \& Osman, A. (1990). Dimensional overlap: Cognitive basis for stimulus-response compatibility-A model and taxonomy. Psychological Review, 97, 253-270. doi:10.1037/0033 $-295 X .97 .2 .253$

MAYR, U. (1996). Spatial attention and implicit sequence learning: Evidence for independent learning of spatial and nonspatial sequences. Journal of Experimental Psychology: Learning, Memory, \& Cognition, 22, 350-364. doi:10.1037/0278-7393.22.2.350

Miller, E. K., \& Cohen, J. D. (2001). An integrative theory of prefrontal cortex function. Annual Review of Neuroscience, 24, 167-202. doi:10.1146/annurev.neuro.24.1.167

Nissen, M. J., \& Bullemer, P. (1987). Attentional requirements of learning: Evidence from performance measures. Cognitive Psychology, 19, 1-32. doi:10.1016/0010-0285(87)90002-8

Olson, I. R., Rao, H., Moore, K. S., Wang, J., Detre, J. A., \& AguirRe, G. K. (2006). Using perfusion fMRI to measure continuous changes in neural activity with learning. Brain \& Cognition, 60, 262-271. doi:10.1016/j.bandc.2005.11.010

PASHLER, H. (1994). Overlapping mental operations in serial performance with preview. Quarterly Journal of Experimental Psychology, 47A, 161-191. doi:10.1080/14640749408401148

PASHLER, H., \& BAYlis, G. (1991). Procedural learning: 1. Locus of practice effects in speeded choice task. Journal of Experimental Psychology: Learning, Memory, \& Cognition, 17, 20-32. doi:10.1037/0278 $-7393.17 .1 .20$

Read, L. E., \& Proctor, R. W. (2004). Spatial stimulus-response compatibility and negative priming. Psychonomic Bulletin \& Review, 11, 41-48.

Reber, A. S., Allen, R., \& Reber, P. J. (1999). Implicit versus explicit learning. In R. J. Sternberg (Ed.), The nature of cognition (pp. 475514). Cambridge, MA: MIT Press.

Reed, J., \& Johnson, P. (1994). Assessing implicit learning with indirect tests: Determining what is learned about sequence structure. Journal of Experimental Psychology: Learning, Memory, \& Cognition, 20, 585-594. doi:10.1037/0278-7393.20.3.585

Remillard, G. (2003). Pure perceptual-based sequence learning. Journal of Experimental Psychology: Learning, Memory, \& Cognition, 29, 581-597. doi:10.1037/0278-7393.29.4.581

Remillard, G. (2009). Pure perceptual-based sequence learning: A role for visuospatial attention. Journal of Experimental Psychology: Learning, Memory, \& Cognition, 35, 528-541. doi:10.1037/a0014646

Richard, M. V., Clegg,, B. A., \& Seger, C. A. (2009). Implicit motor sequence learning is not represented purely in response locations.
Quarterly Journal of Experimental Psychology, 62, 1516-1522. doi:10.1080/17470210902732130

Rowe, J. B., Toni, I., Josephs, O., Frackowiak, R. S. J., \& PassINGHAM, R. E. (2000). The prefrontal cortex: Response selection or maintenance within working memory? Science, 288, 1656-1660. doi:10.1126/science.288.5471.1656

Schmidtke, V., \& Heuer, H. (1997). Task integration as a factor in secondary-task effects on sequence learning. Psychological Research, 60, 53-71. doi:10.1007/BF00419680

Schneider, W., Eschman, A., \& Zuccolotto, A. (2002). E-Prime user's guide. Pittsburgh: Psychology Software Tools, Inc.

Schumacher, E. H., Cole, M. W., \& D'Esposito, M. (2007). Selection and maintenance of stimulus-response rules during preparation and performance of a spatial choice-reaction task. Brain Research, 1136, 77-87. doi:10.1016/j.brainres.2006.11.081

Schumacher, E. H., \& Schwarb, H. (2009). Parallel response selection disrupts sequence learning under dual-task conditions. Journal of Experimental Psychology: General, 138, 270-290. doi:10.1037/ a0015378

Schwarb, H., \& Schumacher, E. H. (2009). Neural evidence of a role for spatial response selection in the learning of spatial sequences. Brain Research, 1247, 114-125. doi:10.1016/j.brainres.2008.09.097

Shanks, D. R., \& Channon, S. (2002). Effects of a secondary task on "implicit" sequence learning: Learning or performance? Psychological Research/Psychologische Forschung, 66, 99-109. doi:10.1007/ s00426-001-0081-2

Stadler, M. A. (1989). On learning complex procedural knowledge. Journal of Experimental Psychology: Learning, Memory, \& Cognition, 15, 1061-1069. doi:10.1037/0278-7393.15.6.1061

Stadler, M. A. (1995). Role of attention in implicit learning. Journal of Experimental Psychology: Learning, Memory, \& Cognition, 21, 674-685. doi:10.1037/0278-7393.21.3.674

Verwey, V. B., \& ClegG, B. A. (2005). Effector dependent sequence learning in the serial RT task. Psychological Research, 69, 242-251. doi:10.1007/s00426-004-0181-x

WillinghaM, D. B. (1999). Implicit motor sequence learning is not purely perceptual. Memory \& Cognition, 27, 561-572.

Willingham, D. B., Nissen, M. J., \& Bullemer, P. (1989). On the development of procedural knowledge. Journal of Experimental Psychology: Learning, Memory, \& Cognition, 15, 1047-1060. doi:10.1037/0278-7393.15.6.1047

Willingham, D. B., Wells, L. A., Farrell, J. M., \& Stemwedel, M. E. (2000). Implicit motor sequence learning is represented in response locations. Memory \& Cognition, 28, 366-375.

ZIESSLER, M. (1998). Response-effect learning as a major component of implicit serial learning. Journal of Experimental Psychology: Learning, Memory, \& Cognition, 24, 962-978. doi:10.1037/0278 $-7393.24 .4 .962$

ZiesSLeR, M., \& NATTKEMPER, D. (2001). Learning of event sequences is based on response-effect learning: Further evidence from a serial reaction task. Journal of Experimental Psychology: Learning, Memory, \& Cognition, 27, 595-613. doi:10.1037/0278-7393.27.3.595

\section{NOTE}

1. The conclusions drawn from Experiment 1 rest primarily on the findings from the RT data. Error data are often inconsistent with the RT data; frequently, when a significant transfer effect is evident in the RT data (indicating sequence learning), no such effect is evident in the error data. One possible explanation for these inconsistencies is that the participant's accuracy was at ceiling. As a result, comparisons in which a significant effect is reported in the error data may be less meaningful than the RT results, and should be interpreted with caution.

(Manuscript received March 2, 2009 revision accepted for publication February 18, 2010.) 